\title{
Modeling and Implementation of Heterogeneous MAS Anti-Missile Battle Sensor Task Planning Based on Community of Interest
}

\author{
Ni Peng ${ }^{*}$, Liu Jin-mang, Wang Gang and Liu Chang-yun
}

\author{
School of Air and Missile Defense, Air force Engineering University, Xi'an, China
}

\begin{abstract}
It is important and difficult for anti-missile battle to use the advantage of resource complementarity of multidimensional sensor platform for collaborative detection and trace. In order to improve the efficiency of mission planning of sensor, for the heterogeneity of the observed resources and the phase and dynamics of missions, the paper introduces the concept of task community, analyzes and establishes heterogeneous MAS multi-sensor task planning system and solution mechanism of problems, based on which the paper focuses on establishing multi-sensor task planning sequence generation model based on cycle-event. And the paper proposes an improved particle swarm optimization algorithm. Simulation experiments indicate that the mechanism established in the paper is rational and effective, and is better than the multisensor task planning effect under traditional mode.
\end{abstract}

Keywords: Multi-sensor task planning, Task community, Heterogeneous MAS, IDPSO.

\section{INTRODUCTION}

Anti-missile battle sensor task planning concerns multidimensional sensor platforms, which mainly includes spacebased satellite and ground-based radar. No matter whether for monitoring area, detection range, detection accuracy or collaborative conduction, there is greater difference between them. The collaborative detection and trace of multidimensional platforms is required in order to fully use the advantage of early-warning force of each dimension [1-5]. The content of anti-missile battle sensor task planning is based on the number, feature and location of the target attacking on ballistic missile to select proper satellite, groundbased or space-based early-warning radar, determine the sequence and time interval of the traced and monitored ballistic missile, and optimize target transition and indication process of multi-dimensional platforms to generate multidimensional sustaining trace ability, which makes the overall efficiency reach the maximum.

Therefore, it is necessary to research the framework of anti-missile sensor task planning technique, and make rational design for the network topology structure and data distribution. The emphasis is to optimize target transition and indication process of multi-dimensional platforms on information processing layer. Under the restriction of limited sensor resources and the constraint of visualized window, the detection sequence, detection time window and operating pattern of sensor for each TBM target is determined dynamically on planning layer, which makes the efficiency optimal.

*Address correspondence to these authors at the School of Air and Missile Defense, Air force Engineering University, Xi'an, China;

Tel: 1986139113; E-mail: Hunter2011@foxmail.com

\section{ESTABLISHMENT OF ANTI-MISSILE BATTLE MULTI-SENSOR TASK PLANNING SYSTEM UNDER HETEROGENEOUS MAS STRUCTURE}

Anti-missile multi-sensor task planning technique is an important guarantee to realize multi-dimensional continuous and stable trace of target. In fact, anti-missile sensor system composes a multi-level and multi-structural network. And multi-dimensional heterogeneous anti-missile sensor platforms consist of the nodes in the network, which is a hybrid large-scale heterogeneous wireless sensor network. Therefore, sensor task planning firstly needs to be based on the establishment of reasonable anti-missile multi-sensor task planning system and demands to be under the overall requirements of optimal network configuration.

\subsection{Architecture of Anti-missile Sensor Collaboration System Based on MAS}

MAS is the research hotspot in DAT field, and is widely applied in supply chain, drone task planning and workshop scheduling [6]. Literature [7] points out that the heterogeneous of the observed resources, and the multi-dimension, phase and dynamics of the observed tasks makes that the modeling of centralized joint task planning method is difficult, the responsibility of solution is high, the robustness of system is worse and the extendibility is insufficient, which requires to use distributive collaboration planning method based on MAS. For ICF generation of NADMS, literature [8] analyzes and compares the system composition, individual behavior and collaborative ability of MAS and NADMS, and proposes that collaboration decision-making method of Agent is effective to implement IFC in MAS. Literature [3] uses MAS to analyze and construct a planning mechanism with distributive collaboration, and uses distributive dynamic planning algorithm to verify the efficacy of the mechanism. 


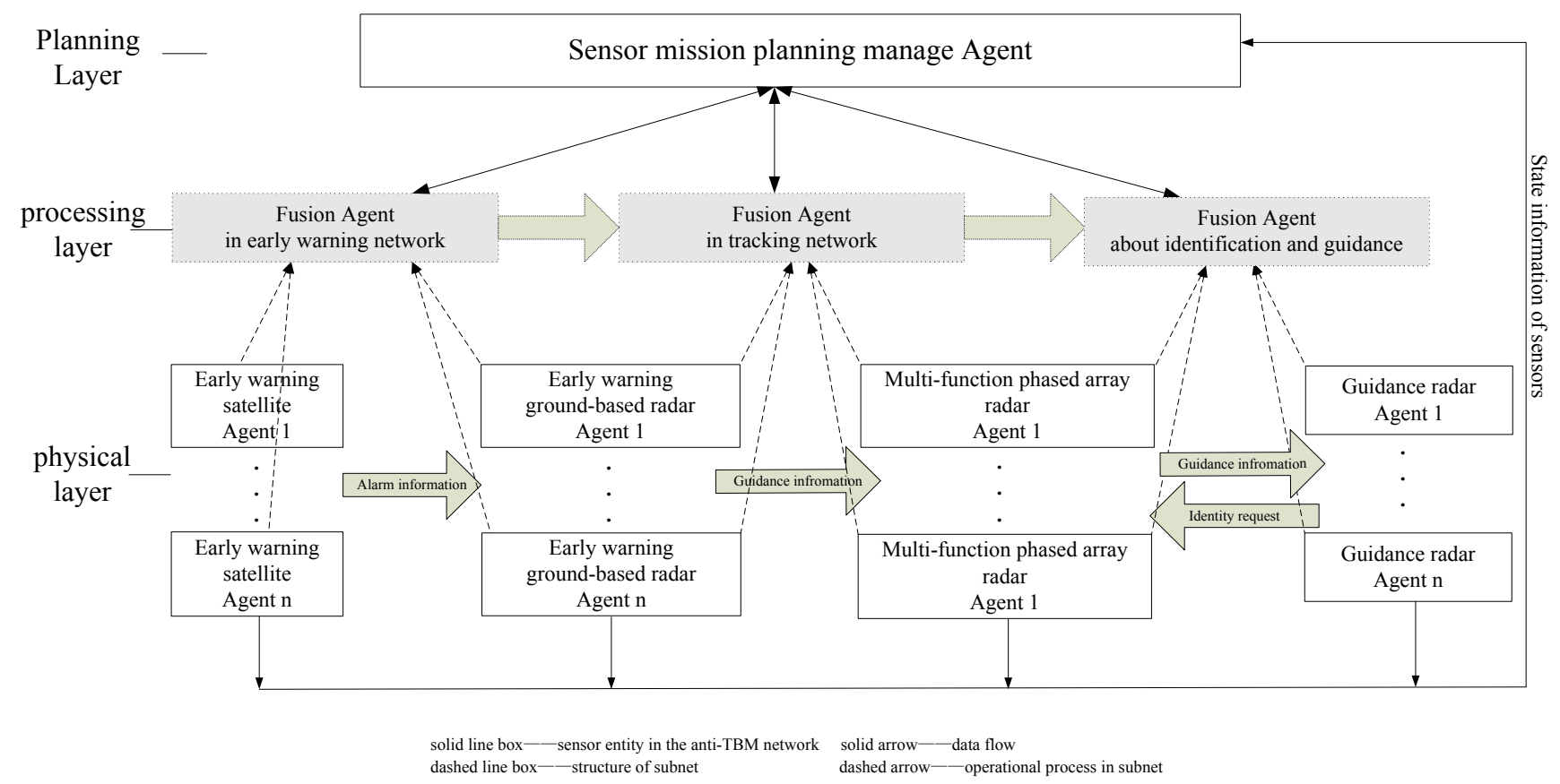

Fig. (1). Architecture of anti-missile battle multi-sensor task planning system based on MAS.

Therefore, the architecture of the constructed anti-missile multi-sensor task planning system based on MAS [9] is shown in Fig. (1).

\subsection{Heterogeneous MAS Multi-Sensor Task Planning Mechanism Based on Community of Interest}

The flight space region of ballistic missile is longer, which breaks away from the view of a satellite or radar to enter the view of another satellite or radar. A specific target only requires some sensor nodes in the anti-missile sensor network for detection rather than needing all nodes in the network. In addition, multi-sensor task planning technique concerns many factors and the collaboration relationship is complicated. Direct global optimization configuration not only can make the dimensions and complicity of problems high, but also is influenced by dynamic adjustment of detection tasks, which makes the robustness of system worse.

Therefore, based on the architecture of the constructed system in Fig. (1), the paper introduces the concept of community of interest, and establishes a new heterogeneous MAS multi-sensor task planning structure and the task planning mechanism. Firstly, the concept of community of interest is introduced.

Community of interest means the collaboration organization or group consisting of the same knowledge background for information exchange, and it is divided into institutional and interest. The former is the presetting configuration, and the latter is the battle alliance which is composed temporarily under distributive network structure.

In anti-missile battle, $\mathrm{COI}$ is the bunching consisting of the dynamics of tasks by a sensor. It consists of all sensor nodes of the detected target in the network. And the bunching sets are from STPs which is made for each threaten target by C2BMC. For the threaten target $t_{i}$, C2BMC stipulates the sensor task planning in ESG according to the mastered situation, $\operatorname{STP}_{i}=\left\{S_{1}, S_{2}, \cdots, S_{n}\right\}$. For time T, COI defines

$$
\begin{aligned}
& \operatorname{COI}_{i}(T)=\left\{t_{i}, S_{j}, S_{k} \cdots S_{t}\right\}, \quad \text { in which } 1<j<k<t<n \\
& {\left[S_{j}, S_{k}, \cdots, S_{t}\right] \subset\left[S_{1}, S_{2}, \cdots, S_{n}\right] .}
\end{aligned}
$$

As shown in Fig. (2), with the promotion of the battle, the attributes of COI consisting of sensor nodes under STPs changes (early-warning--detection--trace--recognition-guidance). The detection range and deployment location of sensors is different, in the flight processes of the targets, the members of COI can be divided into detection state and awaiting relay state, which changes with the time.

\subsection{Description of Battle of Community of Interest}

Based on the idea of community of interest, the paper analyzes and researches anti-missile multi-sensor task planning. The behaviors of community of interest need to be developed to describe their task range and construct the catalogue of community of interest. The formalized descriptions of the ability of three communities of interest are as follows (Fig. 3-5).

\section{GENERATION OF ANTI-MISSILE BATTLE MUL- TI-SENSOR TASK PLANNING SEQUENCE BASED ON CYCLE-EVENT}

Anti-missile battle multi-sensor task planning means to dynamically determine the detection and trace sequence of 


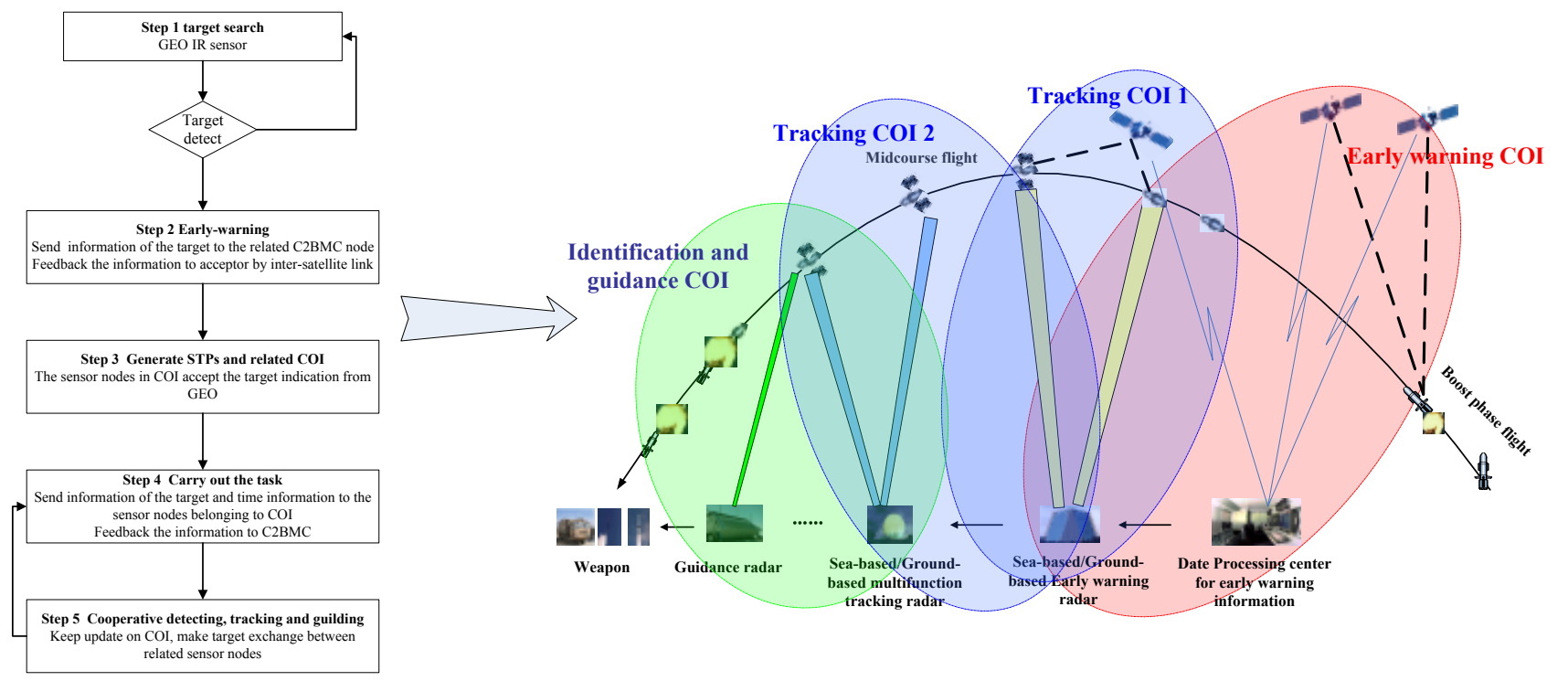

Fig. (2). COI in anti-missile battle multi-sensor task planning process.

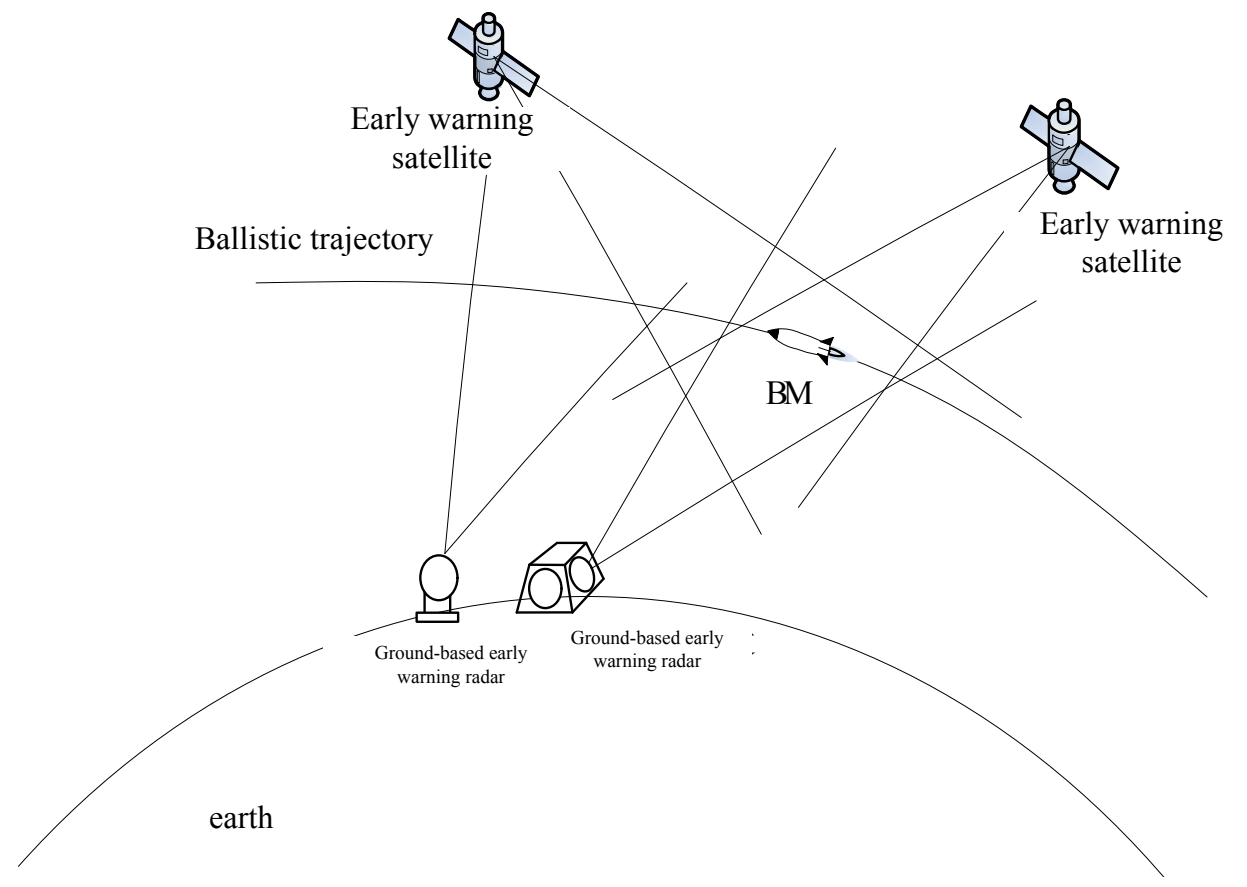

Fig. (3). Example of community of interest of early-warning Agent.

the sensor for the target under the limitation of limited sensor resources and constraint of visualized window, for determining detection time and operation mode and realizing detection, trace and recognition on multiple targets. The essence is a nonlinear combination optimization decision-making problem. As the key problem of operational decision, the scheme and timeliness directly influences the efficacy of weapon system, which has been a research hotpot in military operation field [10]. The integrated strategy based on cycle-event can consider the robustness and adaptation of scheduling scheme, and is the basic strategy to solve the problem.

\subsection{Analysis on Generation Mechanism Based on Com- munity of Interest}

The generation of task planning sequence is dynamic. It not only needs to consider the extensibility of space dimension, but also requires considering efficacy of time dimension. Centralized method is used to generate the general task sequence, for ensuring the present global optimal solution. On the other hand, dynamic events trigger the sensors of the execution layer to make distributive adjustment, for ensuring self-adaptation to dynamic change of tasks. If the generation cycle of the sequence is too long, with the increase of the 


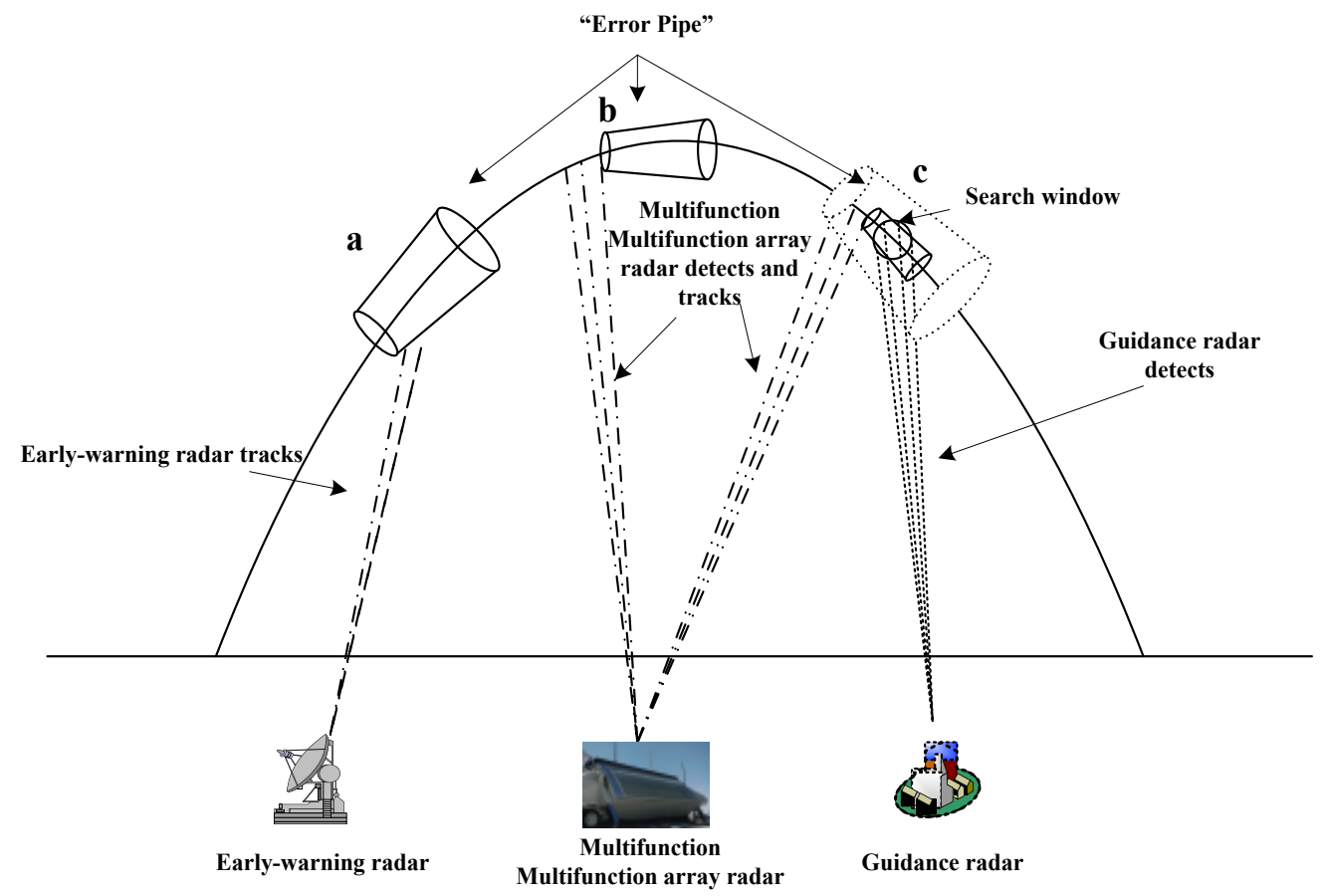

Fig. (4). Example of tracking Agent community of interest.

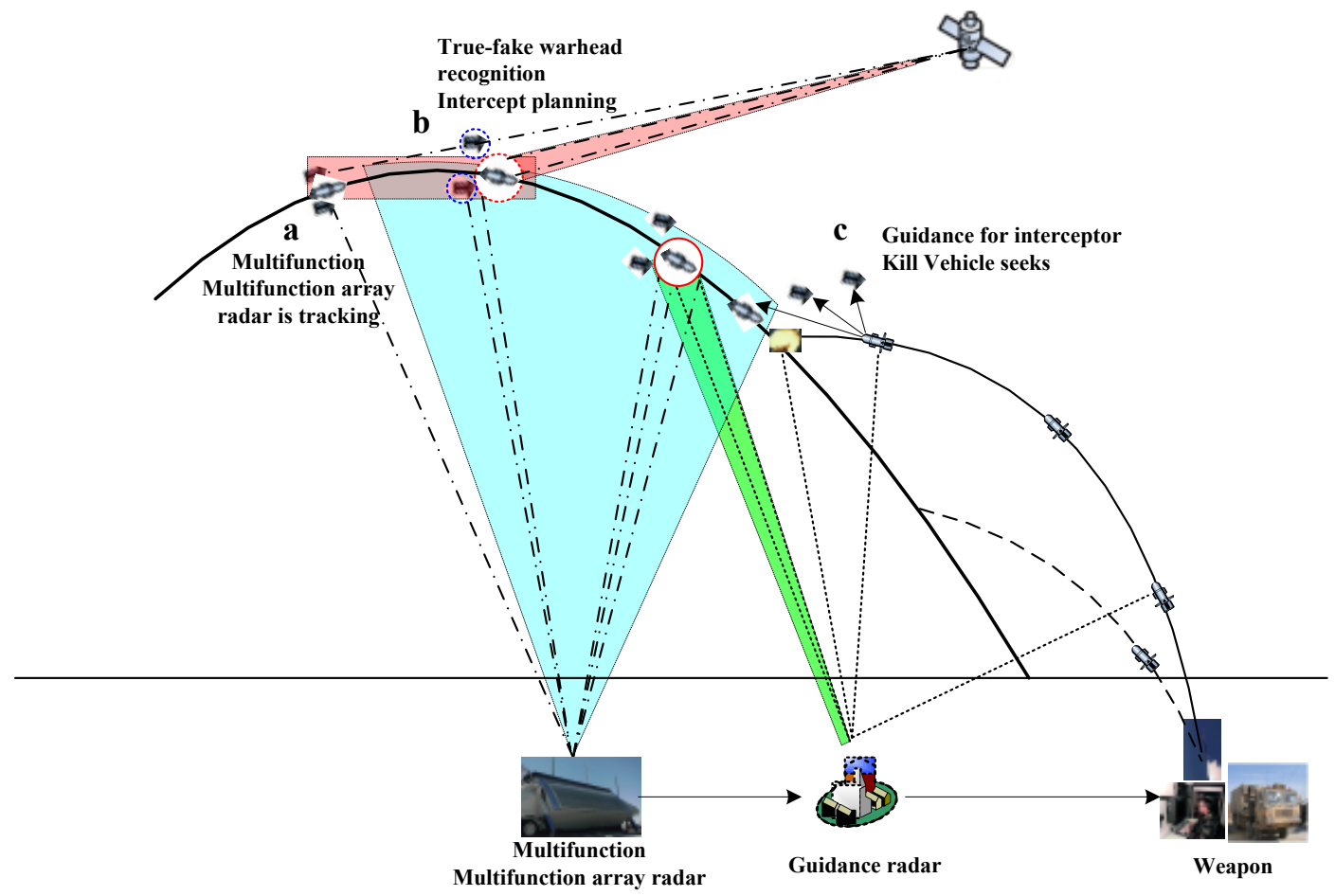

Fig. (5). Example of recognizing and guiding Agent community of interest.

error of detecting and tracking target, planning may fail. Frequent cycle selection evidently increases the workload of community of interest. Therefore, the cycle selection should be adjusted according to the measurement results of the target and the variation tendency of tasks, as shown in Fig. (6).

(1) Determination of cycle
Cyclic sequence generation is to generate new sequences. The selection of cycle has fundamental influence on the robustness of the generation of the sequence scheme. According to the division of the community of interest, the motion trail of the target can be predicted and is stable. Therefore, the community of interest is the basis of the cycle selection. 


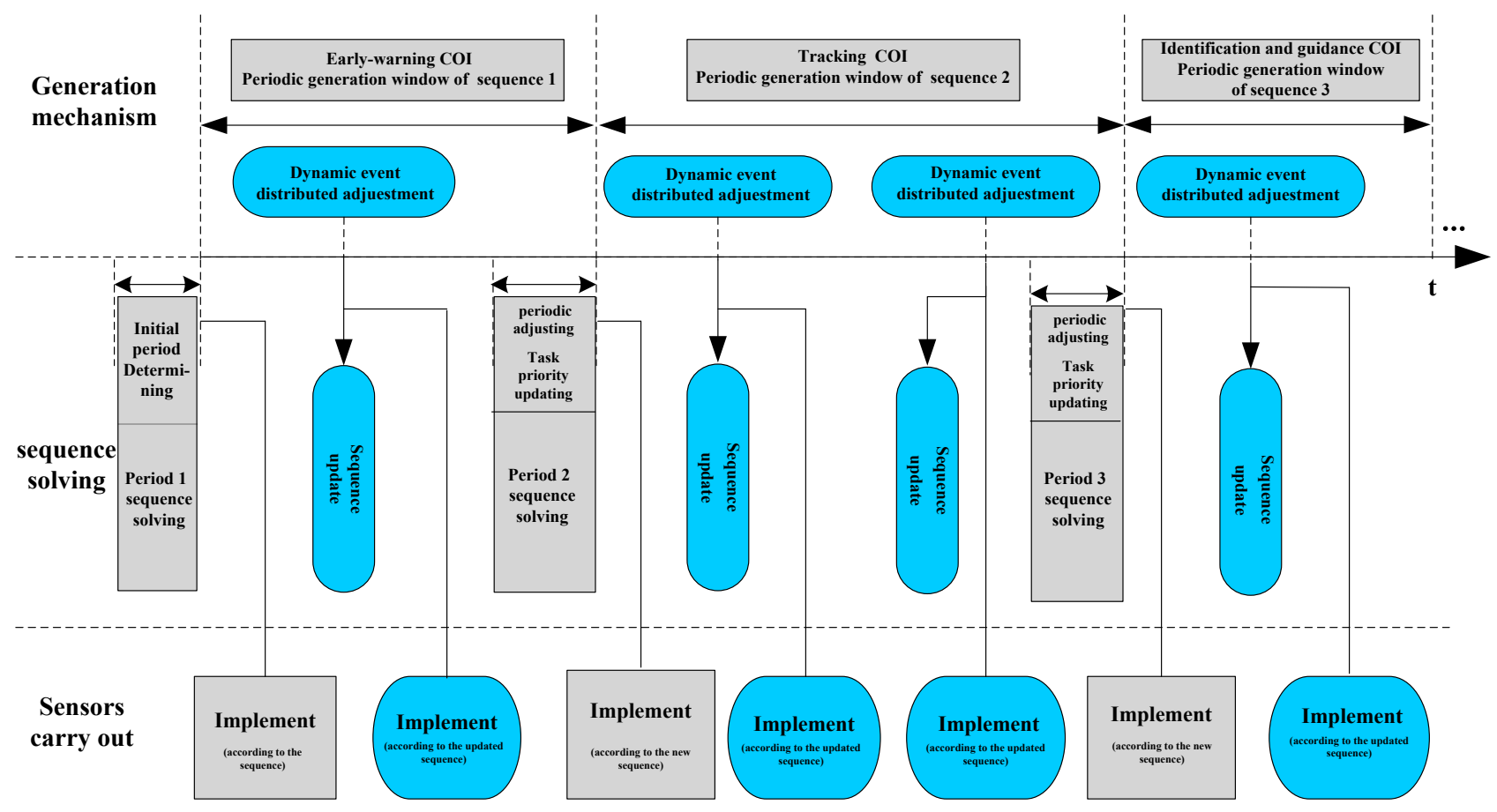

Fig. (6). Generation mechanism of sensor task planning sequence based on cycle-event.

\section{Cycle adjustment based on community of interest}

Cycle adjustment requires to be based on the last execution on the target. As the solution is complicated and it needs to meet the objective requirements, trace accuracy and interceptor determination is selected as two main factors of cycle adjustment for tracing and detecting community of interest and recognizing and guiding community of interest.

$>$ Detecting and tracking community of interest

Detecting and tracking community of interest needs to optimize the tracing performance of the target, so the detection and trace error is selected to determine the time of cycle sequence generation under detecting and tracking community of interest. The initial cycle of the generation of earlywarning community of interest is $T_{0}$, the terminal time of the cycle is $e t$, the threshold of target tracing error is $\varepsilon_{\max }$ and the tracing co-variance for the target under the present community of interest at time $t$ is $P$. T' beginning time of cycle for detecting and tracing community of interest is determined on the basis of a number level higher than the threshold, as shown in formula (1).

$$
\begin{cases}s t^{\prime}=e t, & \left\|P_{t}\right\|>0.1 \varepsilon_{\text {max }} \\ s t^{\prime}=t, & \left\|P_{t}\right\| \leq 0.1 \varepsilon_{\text {max }}\end{cases}
$$

Recognizing and guiding community of interest
Recognition and guidance is the key of online controlling anti-missile battle process, and is the falling point of the sensor task planning. The coupling relationship with interception is necessary to require using successful interception for optimizing the final target. Therefore, interception area is selected to determine the opportunity of generating cycle sequence under recognition and guidance community of task. The information given by trace community of interest can determine the interception point of targets, and the target recognition window can be used to achieve the objective of optimizing sequences.

The time-location parameter of interception point is $\left(t_{i H i t}, x_{i H i t}, y_{i H i t}, z_{i H i t}\right)$, and the time-location parameter of target is $\left(t_{\text {iLaun }}, x_{\text {iLaun }}, y_{\text {iLaun }}, z_{\text {iLaun }}\right)$. The time from the firing location of the target to the interception point is $\Delta t_{1}$, and recognition time window is $\Delta t_{2} \cdot t_{\text {iTrack }}$ which means that the trace and guidance radar finds the target is the distance of discovering the target, which determines the starting distance of trace and guidance radar beginning to trace. So scheduling opportunity is as shown in the formula (2).

$T_{\text {ident }}=\left[t_{\text {iTrack }}, t_{\text {iLaun }}\right]$

Only successfully recognizing the target during the period can achieve the launch conditions of targets. 


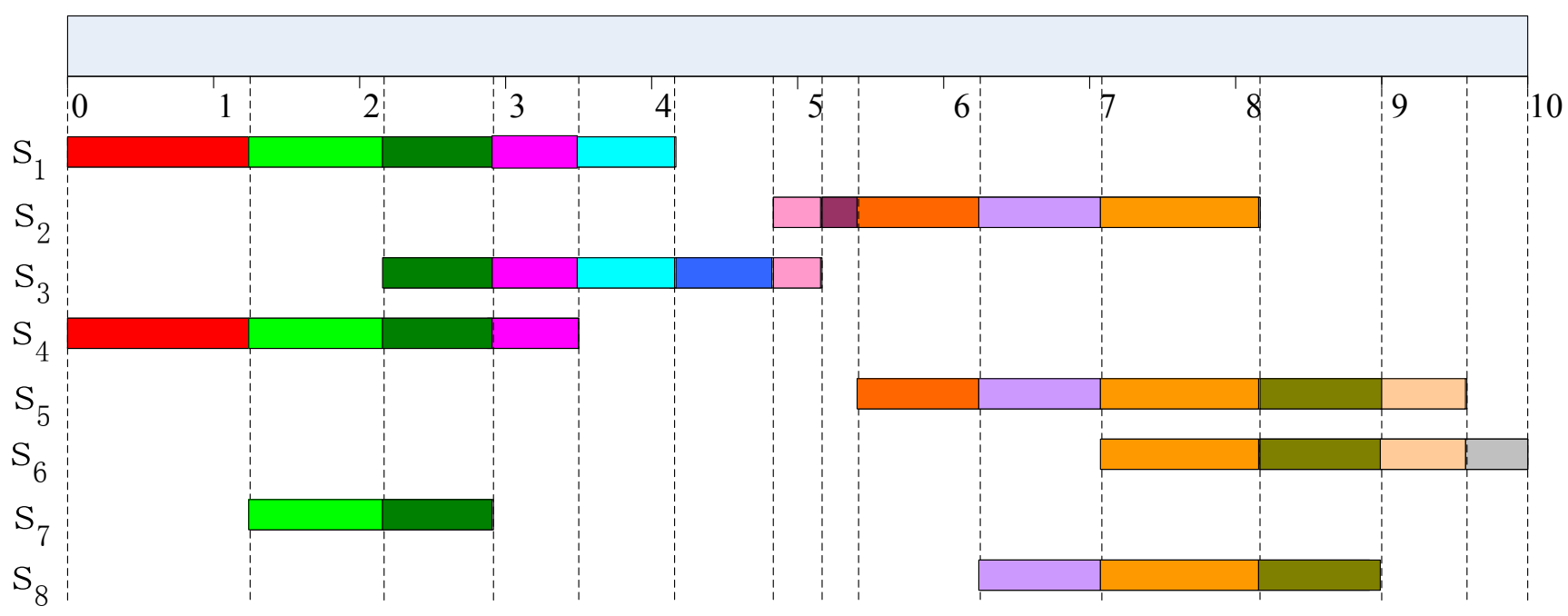

Fig. (7). Task decomposition strategy based on beginning and ending time.

Distributive update

Distributive update needs to design collaboration mechanism which matches with the problems. The allocation nodes use the collaboration mechanism as the behavior rule, and the allocation scheme receives dynamic and distributive adjustment. The core of solving the problem is to design collaboration mechanism of task performers. And the performers use the collaboration mechanism as the behavior rule for realizing dynamic update. The method in literature (Peng Ni. et al, 2014) is used for distributive update on COI sequence.

\subsection{Modeling of Cyclic Sequence Generation}

(1) Task decomposition

In order to reduce the complicity of interactive relationship of the original problem, there is need to decompose the life cycle of the tasks into continuous or discrete times according to the beginning time and the ending time of visualized time window of sensor and target. And the decomposed time corresponds to a sub-task. The paper uses task decomposition strategy based on beginning-ending time (Ren junlliang. et al, 2014), and the task after decomposition is called elementary task. The cycle [0-10] is taken as an example, and the task decomposition is shown in Fig. (7).

In a cycle, the set of all tasks attacking the target is $T A=\left\{T_{i} \mid i=1,2, \cdots m\right\}$, and it defines the task on target $i$,

$T_{i}=\left\{S T_{i}, E T_{i}, T p r_{i}, R S_{i}, S N_{i}, E r_{i}\right\}$

$S T_{i}$ means the beginning time of the task, and $E T_{i}$ means the tending time of the task. $T P R_{i}$ means the present priority of the task has no relationship with the feature of the task and the importance of the target. And recognizing task is they to anti-missile battle, so task priority is generally the highest. $R S_{i}$ is the set of sensors which have visual relationship with tasks. $S N_{i}$ means the number of elementary tasks after decomposition, and $E r_{i}$ means the present information error of the task.

The $j$ elementary task $P T_{i, j}$ of $T_{i}$ after decomposition is defined as follows.

$$
P T_{i, j}=\left\{S T_{i, j}, E T_{i, j}, \operatorname{Pr}_{i, j}, R S_{i, j}, R N_{i, j}, E r_{i, j}, \operatorname{Dis}_{i, j}^{l}, \operatorname{Pow}_{i, j}^{l}\right\}
$$

$S T_{i, j}$ means the beginning time of the elementary task, and $E T_{i, j}$ means the ending time. $\operatorname{Pr}_{i, j}$ means the priority of the elementary task. $R S_{i, j}$ means the set of the sensors of elementary task, and there are $R N_{i, j}$, in which the $l$ sensor is represented as $R S_{i, j}^{l}$. $E r_{i, j}$ is the information error o the elementary task. $D i s_{i, j}^{l}$ means the average distance of sensor nodes and target while executing elementary tasks. $P o w_{i, j}^{l}$ is the resource consumed by sensor node $l$ executing task.

(2) Planning model

The subscript, parameter and decision-making parameters of the model are shown in Table $\mathbf{1 .}$

The objective of anti-missile battle sensor task planning is to optimize resource distribution: 1. Maximizing detection favorable degree. 2. Minimizing sensor nodes. 3. Balancing global resource load. So the target function is formula (3).

$$
\max _{x} F(x)=\lambda_{1} A(x)+\lambda_{2} B(x)-\lambda_{3} C(x)
$$


Table 1. Description of symbols in the model.

\begin{tabular}{|c|c|c|c|}
\hline Symbol & Definition & Symbol & Definition \\
\hline$I$ & Number set of incoming targets & \multirow{2}{*}{$\rho_{e}$} & \multirow{2}{*}{$\begin{array}{l}\text { Deviation of detection angle of sensor target and } \\
\text { the best detection angle at } E T_{i, j}\end{array}$} \\
\hline$M$ & Number set of sensors & & \\
\hline$A(x)$ & Detecting favorable factors & $\theta_{o p t}^{l}$ & The best detection angle of the sensor $l$ \\
\hline$B(x)$ & $\begin{array}{l}\text { Resource utilization efficiency factor (switching } \\
\text { time) }\end{array}$ & $\mathbf{r}_{s}^{l}\left(S T_{i, j}\right)$ & Vector from sensor $l$ to target i at $S T_{i, j}$ \\
\hline$C(x)$ & Global load balance factor & $\mathbf{r}_{e}^{l}\left(E T_{i, j}\right)$ & Vector from sensor $l$ to target i at $E T_{i, j}$ \\
\hline$x_{i, j}^{l}$ & $\begin{array}{c}\text { Decision variable, } x_{i, j}^{l}=1 \text { means that the sensor } l \\
\text { executes elementary task } S T_{i, j}, \text { or } x_{i, j}^{l}=0\end{array}$ & $\mathbf{v}_{s}^{l}\left(S T_{i, j}\right)$ & Velocity vector of sensor $l$ for target $i$ at $S T_{i, j}$ \\
\hline$T p r_{i}$ & Task priority of incoming target $i$ & $\mathbf{v}_{e}^{l}\left(E T_{i, j}\right)$ & Velocity vector of sensor $l$ for target $i$ at $E T_{i, j}$ \\
\hline$\left[\right.$ Twin $\min _{i}^{l}$, Twin $\left.\max _{i}^{l}\right]$ & $\begin{array}{l}\text { Visualized window of the sensor } l \text { for the incoming } \\
\text { target } i\end{array}$ & $S W T_{\max }^{l}$ & $\begin{array}{l}\text { The maximal time of sensor } l \text { for target conver- } \\
\text { sion }\end{array}$ \\
\hline$D \max ^{l}$ & The maximal operating distance of the sensor 1 & $S W T_{i 1, i}^{l}$ & $\begin{array}{l}\text { Time for the conversion of sensor } l \text { from target } i 1 \\
\text { to target } i\end{array}$ \\
\hline$\rho_{s}$ & $\begin{array}{l}\text { The deviation of detection angle of sensor target at } \\
\qquad S T_{i, j} \text { and the best detection angle }\end{array}$ & & \\
\hline
\end{tabular}

$$
\begin{aligned}
& A(x)=\max \left\{\sum_{i \in I} T h r_{i}\left[\sum_{j \in S N_{i}} \sum_{l \in R N_{i, j}} \cdot \begin{array}{c}
x_{i, j}^{l} \cdot \frac{T w \operatorname{wanax}_{i}^{l}-S T_{i, j}}{E T_{i, j}-S T_{i, j}} \\
\cdot\left(\frac{D \max ^{l}-D i s_{i, j}^{l}}{D \max ^{l}}+\frac{\pi-\left(\rho_{s}+\rho_{e}\right)}{\pi}\right)
\end{array}\right]\right\} \\
& B(x)=\max \left\{\sum_{i \in I} \sum_{j \in S N_{i}} \sum_{l \in R N_{i, j}} x_{i, j}^{l} \cdot \frac{S W T_{\max }^{l}-S W T_{i l, i}^{l}}{S W T_{\max }^{l}}\right\} \\
& \left\{C(x)=\min \left\{\sum_{i \in I} \sum_{j \in S N_{i}} \sum_{l \in R N_{i, j}} \frac{x_{i, j}^{l}}{T_{i} \cdot S N_{i, j}}\right\}\right. \\
& \rho_{s}=\left|\theta_{o p t}^{l}-\arccos \frac{\mathbf{r}_{s}^{l}\left(S T_{i, j}\right) \cdot \mathbf{v}_{s}^{l}\left(S T_{i, j}\right)}{\mid \mathbf{r}_{s}^{l}\left(S T_{i, j}\right) \cdot \mathbf{v}_{s}^{l}\left(S T_{i, j}\right)}\right| \\
& \rho_{e}=\left|\theta_{o p t}^{l}-\arccos \frac{\mathbf{r}_{e}^{l}\left(E T_{i, j}\right) \cdot \mathbf{v}_{e}^{l}\left(E T_{i, j}\right)}{\mid \mathbf{r}_{e}^{l}\left(E T_{i, j}\right) \cdot \mathbf{v}_{e}^{l}\left(E T_{i, j}\right)}\right| \mid
\end{aligned}
$$

Formula (4) means to maximize target detection favorable degree, and $T p r_{i}$ considers the threat degree of target and property of tasks (when it is recognition and guidance task, there is the task feature without allowing interruption, and $T p r_{i}>0.9$ ). Formula (5) represents maximizing resource utilization efficiency. The grater the value, the less the influence of sensor conversion on continuous detection, and the higher the resource utilization is. Formula (6) means the total load rate of balancing sensor resource. In order to ensure the load can correspond to emergencies in balance, for a cycle sequence, the smaller the $C(x)$, the better it is. Formula (7) and formula (8) mean the influencing degree of detection angle on accuracy of sensor tracing targets at $S T_{i, j}$ and $E T_{i, j}$ respectively. The anti-missile battle multi-sensor task planning sequence generation model is established, as follows.

$$
\begin{aligned}
& \max \quad F(x)=\sum_{i \in I} \sum_{j \in S N_{i}} \sum_{l \in R N_{i, j}} x_{i, j}^{l} \cdot c_{i, j}^{l} \\
& c_{i, j}^{l}=\lambda_{1} \frac{T w i n \max _{i}^{l}-S T_{i, j}}{E T_{i, j}-S T_{i, j}} \cdot\left(\frac{D \max ^{l}-D i s_{i, j}^{l}}{D \max ^{l}}+\frac{\pi-\left(\rho_{s}+\rho_{e}\right)}{\pi}\right) \\
& +\lambda_{2} \frac{S W T_{\max }^{l}-S W T_{i 1, i}^{l}}{S W T_{\max }^{l}}-\lambda_{3} \frac{x_{i, j}^{l}}{T_{i} \cdot S N_{i, j}} \\
& \left\{\sum_{l \in\left\{x \mid x \in R S \lambda_{1}+R S_{i, j}\right\}}^{\lambda_{1}+\lambda_{2}+\lambda_{3}=1} x_{i, j}^{l}=0\right. \\
& S W T_{\max }^{l} \geq S W T_{i, j}^{l} \\
& \text { S.t }\left\{\text { Pow }_{\max }^{l}-\sum_{i \in I} \sum_{j=1}^{S N_{i}} x_{i, j}^{l} \cdot \operatorname{Pow}_{i, j}^{l} \geq 0\right. \\
& \text { if }\left(T p r_{i} \geq 0.9\right), \prod_{j=1}^{S N_{i}} x_{i, j}=1 \\
& \text { if }\left(E T_{i 1, j 1}-S T_{i 2, j 2}\right)>0, x_{i 1, j 1}^{l}+x_{i 2, j 2}^{l} \leq 1
\end{aligned}
$$

Formula (9) means to maximize the profits of sensors completing all tasks in a cycle, and $x_{i, j}^{l}$ is a decision variable. If the sensor $l$ executes elementary task $S T_{i, j}, x_{i, j}^{l}=1$, or 
$x_{i, j}^{l}=0$. If there are $n$ sensor nodes and $m$ missiles, the decision matrix of all elementary task of the task $T_{i}$ is

$$
X_{i}=\left[\begin{array}{ccc}
x_{i, 1}^{1} & \cdots & x_{i, S N_{i}}^{1} \\
\vdots & \ddots & \\
x_{i, 1}^{n} & \cdots & x_{i, S N_{i}}^{n}
\end{array}\right]
$$

The decision matrix of all tasks is shown in formula (18). Line number represents the number of sensor nodes, and column number means the number of elementary tasks.

$$
\begin{aligned}
X & =\left[X_{1}, X_{2}, \cdots X_{m}\right]=\left[\begin{array}{cccccccccc}
x_{1,1}^{1} & \cdots & x_{1, S N_{i}}^{1} & x_{2,1}^{1} & \cdots & x_{2, S N_{i}}^{1} & \cdots & x_{m, 1}^{1} & \cdots & x_{m, S N_{i}}^{1} \\
\vdots & \ddots & \vdots & \vdots & \ddots & \vdots & \ddots & \vdots & \ddots & \vdots \\
x_{1,1}^{n} & \cdots & x_{1, S N_{i}}^{n} & x_{2,1}^{n} & \cdots & x_{2, S N_{i}}^{n} & \cdots & x_{m, 1}^{n} & \cdots & x_{m, S N_{i}}^{n}
\end{array}\right] \\
& =\left[\begin{array}{cccccc}
x_{11}^{1} & \cdots & x_{1, S N_{i}+1}^{2} & \cdots & x_{1, \Sigma_{i e n}^{m} S N_{i}}^{m} \\
\vdots & \ddots & \vdots & \ddots & \vdots \\
x_{n 1}^{1} & \cdots & x_{1, S N_{1}+1}^{2} & \cdots & x_{n, \sum_{i e m}^{m}}^{m} S N_{i}
\end{array}\right]
\end{aligned}
$$

Formula (10) means that the total benefits value of sensor $l$ executing elementary task $P T_{i, j}$. The conversion method of decision matrix $X$ can be used to get the profit matrix $\boldsymbol{C}_{i}$ of all sensors for target $i$. In the cycle, the profit matrix of $n$ targets is $\boldsymbol{C}=\left[\boldsymbol{C}_{1}, \boldsymbol{C}_{2}, \ldots, \boldsymbol{C}_{n}\right]$. Formula (11) is the weight constraint, which can be achieved by simulation analysis or historical experience. Formula (12) means elementary task $P T_{i, j}$ sensor resource constraint, which only can be selected between the sensors of visualized time window. Formula (13) means the time constraint of sensor converting targets. Formula (14) represents the energy constraint of sensor resources in a cycle sequence. Formula (15) means not to allow interrupting task constraint. When the priority is greater than 0.9 , the task for target $i$ is not to allow interruption. Formula (16) means that the subtasks with time interleaving can't use the same sensor resource.

\subsection{Solution Algorithm for Model}

The paper focuses on verifying the rationality and feasibility of the established framework mechanism, and selecting an easy and common algorithm as the solution. PSO algorithm has the characteristics of easy operation, few adjustable parameters and rapid convergence [11-13]. An improved DPSO algorithm is introduced as follows.

\section{(1) Particle coding}

The paper uses equivalence to represent disperses binary coding method to optimize parameters. The decision matrix $\mathrm{X}$ is decomposed and converted into one-dimensional row vector. Each variable of the matrix corresponds to the components of row vector of the converted row vector. For example, the decision matrix is $\left[x_{11}^{1}, x_{12}^{1}, x_{13}^{2}, x_{14}^{2} ; x_{21}^{1}, x_{22}^{1}, x_{23}^{2}, x_{24}^{2}\right]$, the converted row vector based on column decomposition is $\quad\left[x_{11}^{1}, x_{21}^{1}, x_{12}^{1}, x_{22}^{1}, x_{13}^{2}, x_{23}^{2}, x_{14}^{2}, x_{24}^{2}\right]$ If $\left[x_{11}^{1}, x_{21}^{1}, x_{12}^{1}, x_{22}^{1}, x_{13}^{2}, x_{23}^{2}, x_{14}^{2}, x_{24}^{2}\right]=[10010110]$, it means that the sensor node 1 of the first elementary task of the target 1 executes and the sensor node 2 doesn't execute, and the second sensor node 2 of the first elementary task of the target 2 executes. In addition, the sensors of targets can be tracked and decomposed into many virtual single-tracking sensor resources. The structure not only can directly represent all solutions, but also ensures that the number of targets distributed by each sensor is less than the capacity.

\section{(2) Initialization}

In a $n$-dimensional search space, there is a population consisting of $n$ particles, $X=\left\{x_{1}, x_{2}, \ldots, x_{m}\right\}$. The position vector of the $i$ particle is $x_{i}=\left[x_{i 1}, x_{i 2}, \ldots, x_{i n}\right]$, the velocity vector is $v_{i}=\left[v_{i 1}, v_{i 2}, \ldots, v_{i n}\right]$, the optimal position searched by the $i$ particle is $p_{\text {ibest }}=\left[p_{i 1}, p_{i 2}, \ldots, p_{i n}\right]$, and the optimal position searched by all particles is $p_{g b e s t}=\left[p_{g 1}, p_{g 2}, \ldots, p_{g n}\right]$.

The following steps are taken to generate particles.

Step 1. Particle component $x_{i m}^{j}$ is randomly assigned with 0 or 1 .

Step 2. If $x_{i m}^{j}=1$, it needs to be judged if it and other particle components meet the constraint of formula (16). If it meets, turning to Step 5, or turning to Step 3 ;

Step 3. According to $T p r_{i}$ of elementary task during overlapping period, the sensors are selected in the descending order. If there is no sensor being selected, the particles are deleted.

Step 4. According to the relationship of task and sensor in Step 3, the initial particles are updated, and turning to Step 5.

Step 5. Output feasible particle coding.

(3) Particle update

The velocity of particle, $v_{i d}$ and $x_{i d}(d=1,2, \ldots, n)$, is adjusted according to the following formula.

$v_{i d}^{(t+1)}=\omega v_{i d}^{(t)}+c_{1} r_{1}\left(p_{i d b e s t}^{(t)}-x_{i d}^{(t)}\right)+c_{2} r_{2}\left(p_{\text {gbest }}^{(t)}-x_{i d}^{(t)}\right)$

$x_{i d}^{(t+1)}=x_{i d}^{(t)}+v_{i d}^{(t+1)}$

In the formula, $t$ is the present evolution algebra, $\omega$ is the inertia factor, and $c_{1}$ and $c_{2}$ are the learning factors.

$x_{i m}^{j}$ of the coding is 0 or 1 , so PSO needs to be transformed. If $x_{i d}^{(t+1)}$ exceeds the value range, the following formula is used for update. 


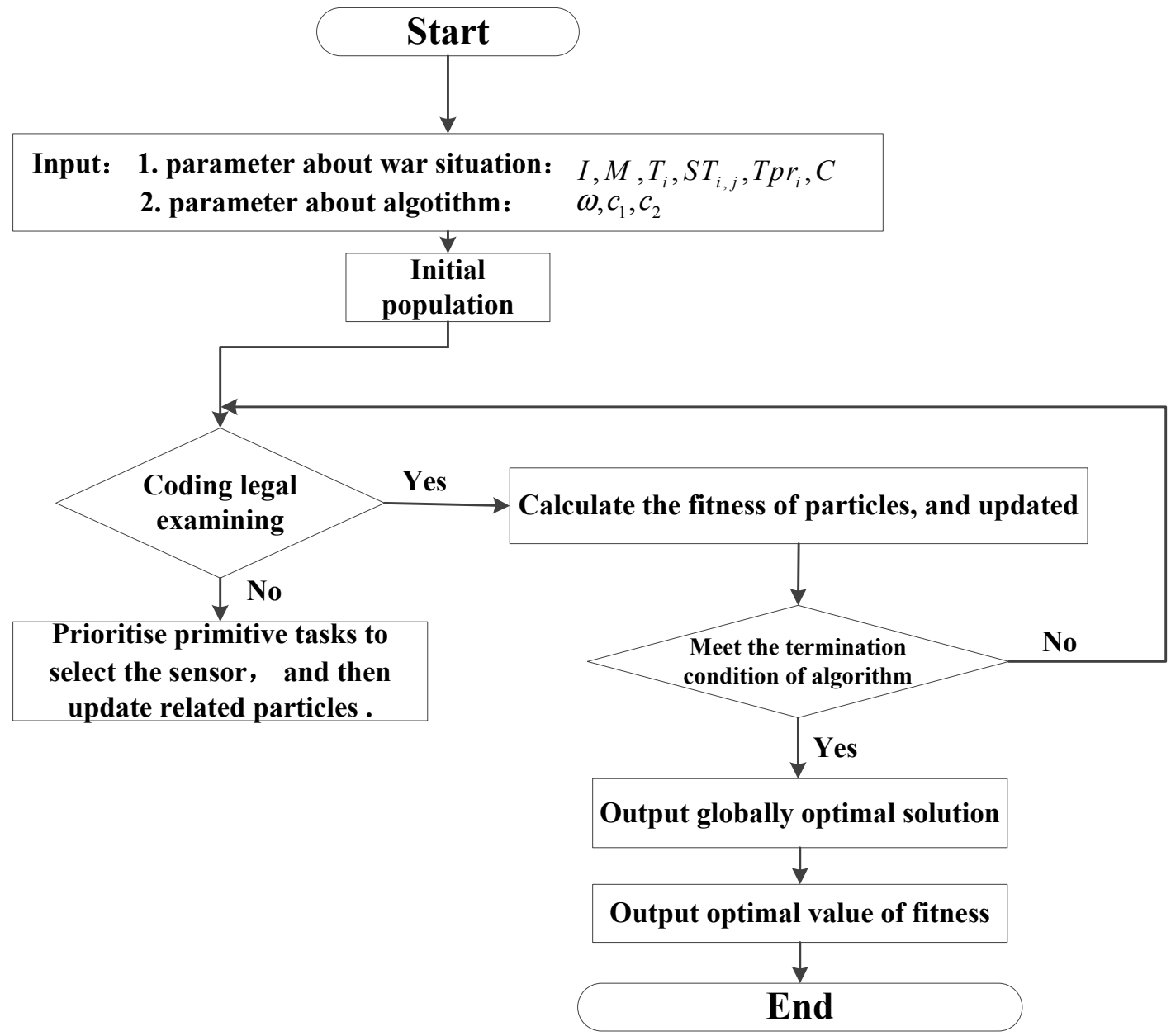

Fig. (8). Algorithm flow.

$\begin{cases}x_{i d}^{(t+1)}=x_{i d}^{(t+1)}+\omega^{*} \text { rand }^{*}\left[x_{i \max }-x_{i \min }\right] & x_{i d}^{(t+1)}<x_{i \min } \\ x_{i d}^{(t+1)}=x_{i d}^{(t+1)}-\omega^{*} \text { rand }^{*}\left[x_{i \max }-x_{i \min }\right] & x_{i d}^{(t+1)}>x_{i \max }\end{cases}$

BPSO algorithm proposed by Kennedy and Eberhart is used. The principle is to limit each dimension of $x_{i}, p_{i}$ and $p_{g}$ to be 0 or 1 , and the velocity uses Sigmoid function

$S(v)=\frac{1}{1+e^{-v}}$

To represent the possibility of change, the velocity is the same with formula (19). The position updating process is

If $r_{0}<S\left(v_{i d}^{(t+1)}\right) x_{i d}^{(t+1)}=1$. If $r_{0} \geq S\left(v_{i d}^{(t+1)}\right) x_{i d}^{(t+1)}=0$.

(4) Algorithm flow

For the actual timeliness in the battle, the terminal condition based on iterations is used, and the algorithm can give satisfying solution in acceptable time range. The overall algorithm flow is shown in Fig. (8).

\section{SIMULATION AND VERIFICATION SIMULATION SCENARIO}

In order to simplify experiments for verifying the rationality and efficacy of the framework mechanism of the system, the following scenarios are introduced, three DSP satellites with geosynchronous orbit, 5 multi-function phased array radars, one long-distance early-warning radar and two ballistic targets. The performance parameters in literature [11] are used for mathematical modeling. IDPSO algorithm controls parameter setting. The scale of particle swarm is 100 , and $w=1, c_{1}=c_{2}=2$ algorithm terminal condition is that the maximal iterations is 100 . The mechanism of the paper and traditional is used for simulation analysis, as shown in Fig. (9).

\subsection{Results and Analysis}

The coordinate curve of two missiles for each radar achieved in the simulation is shown in Fig. (10) to Fig. (13).

The method in the paper is compared with the operation mode under traditional structure, and the operation results are shown in Table 2. 


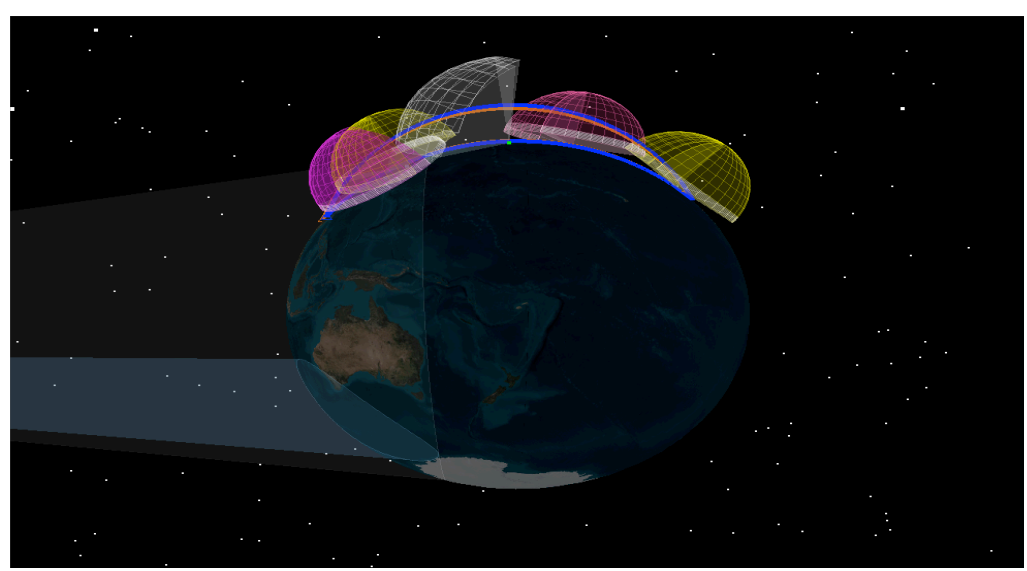

(a) Deploy scenario

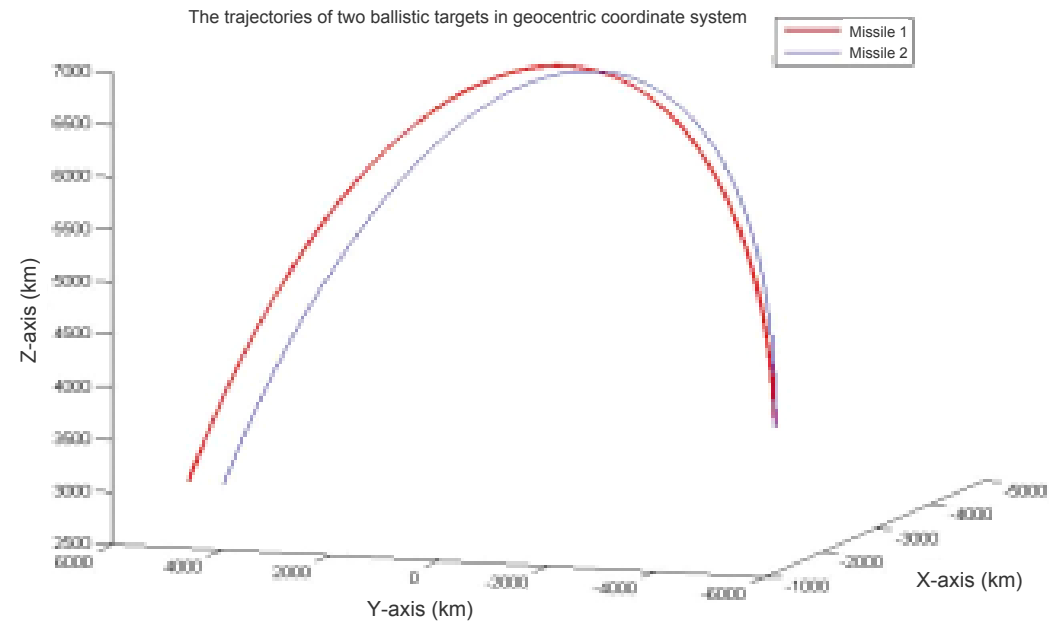

(b) TBM ballistic feature simulation curve

Fig. (9). War-situation scenario setting.

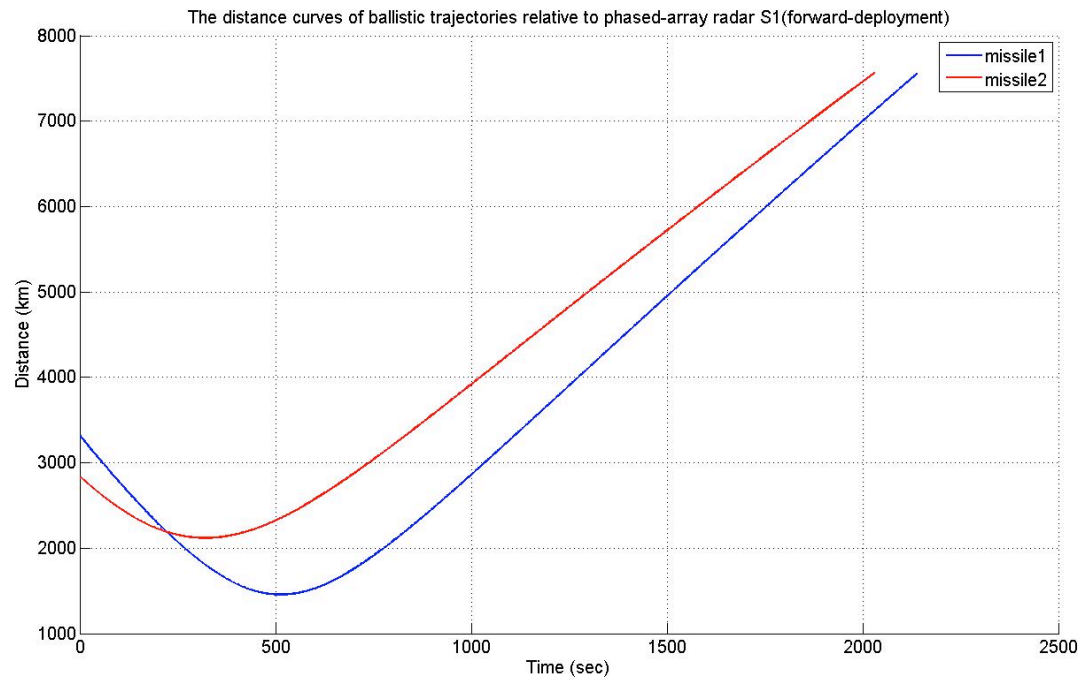

(a) Distance curves

Fig. (10). Contd... 


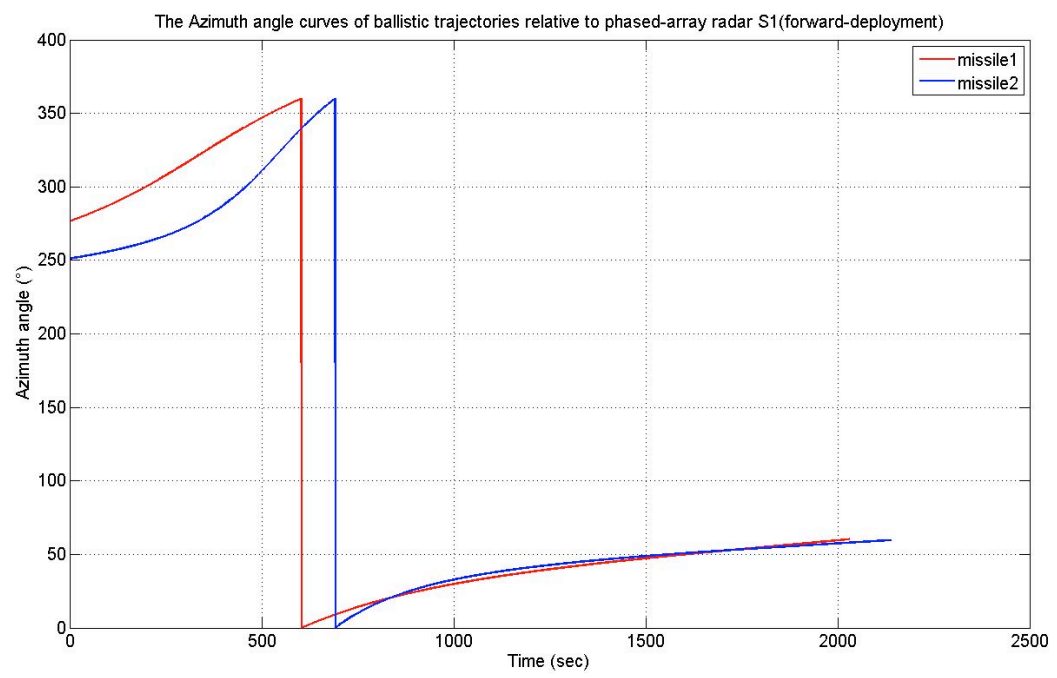

(b) Azimuth angle curves

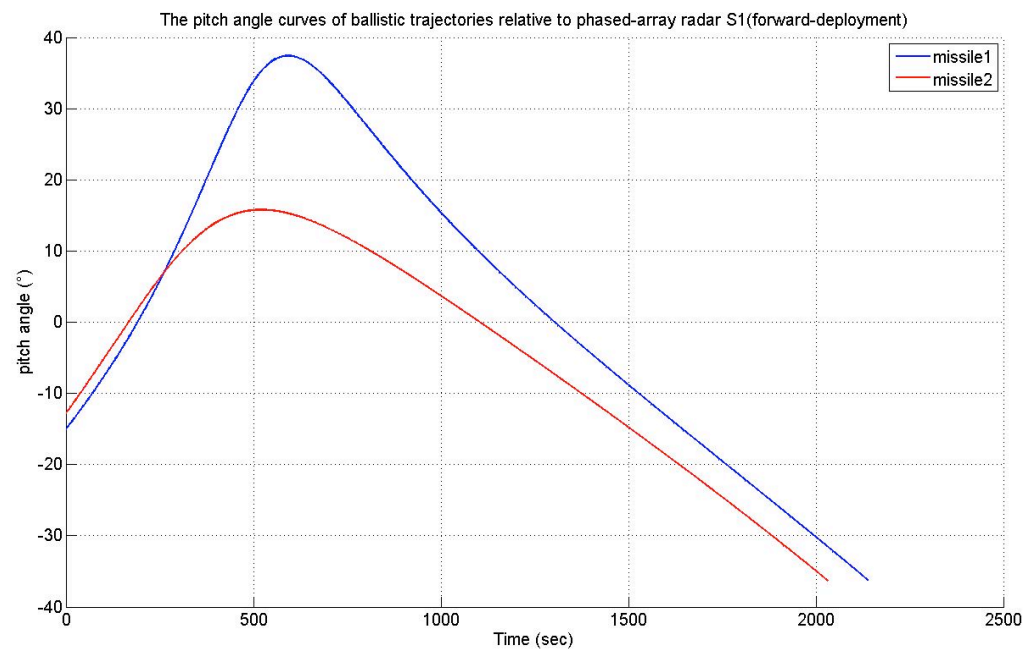

(c) Pitch angle curve

Fig. (10). The coordination curves of ballistic trajectories relative to phased-array radar $S_{l}$ (forward- deployment).

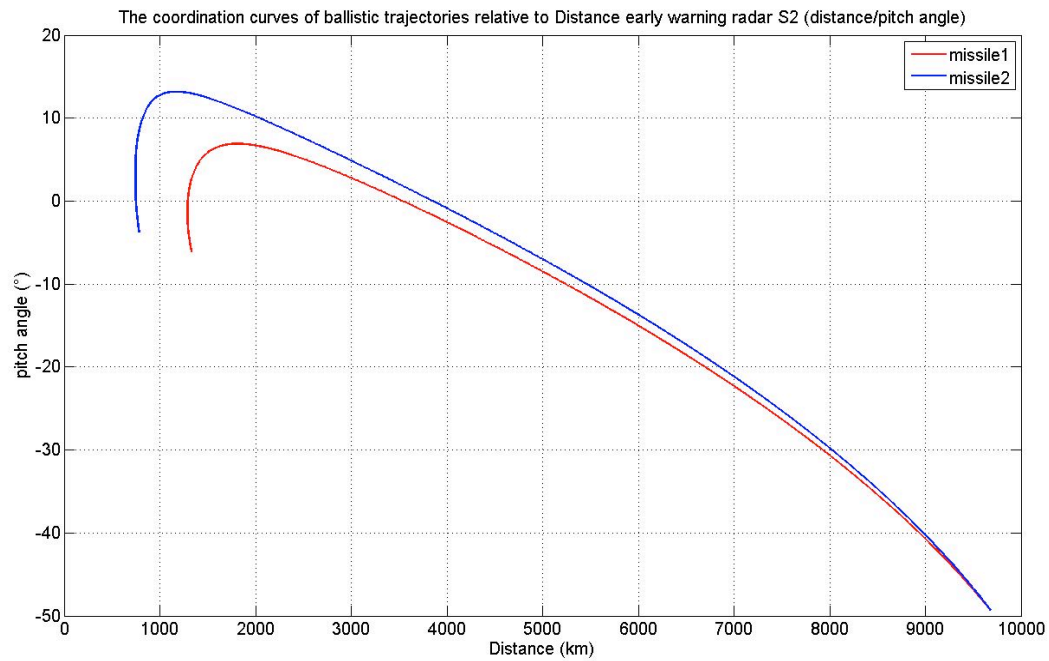

Fig. (11). The coordination curves of ballistic trajectories relative to Distance early trajectories relative to warning radar S2 (distance/pitch angle). 


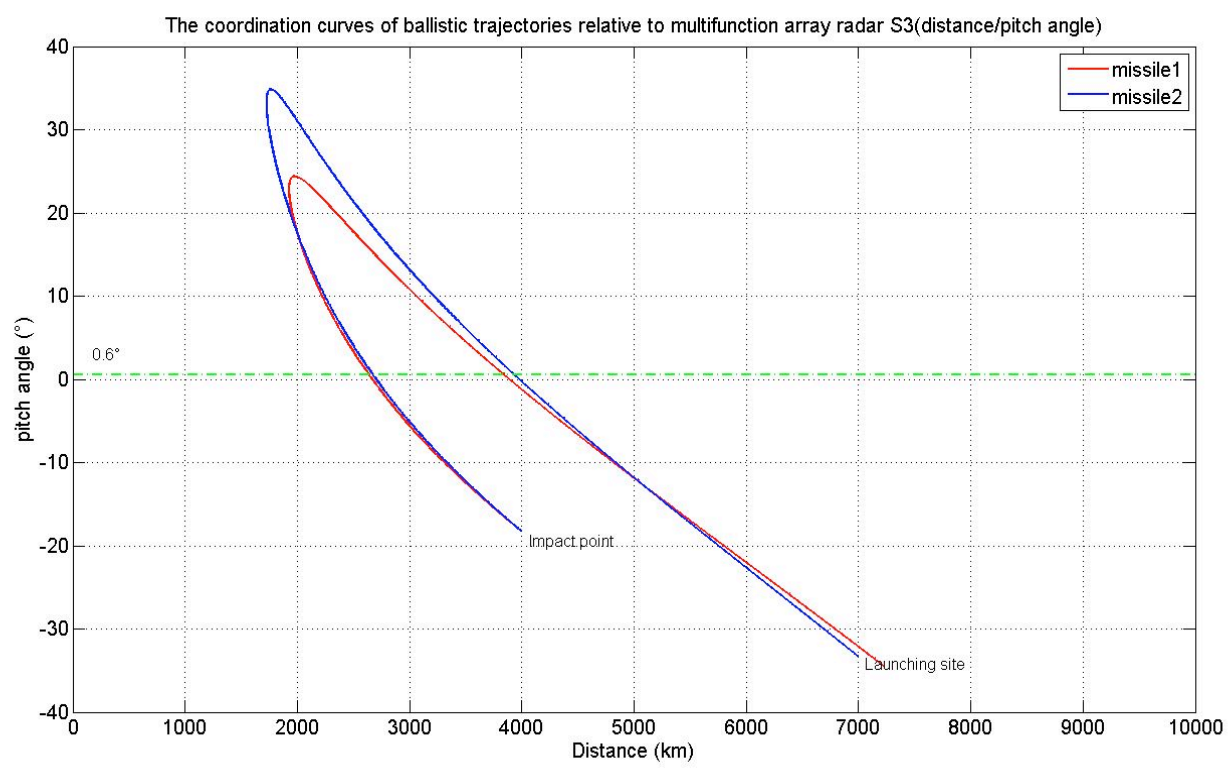

(a) Distance/Pitch angle

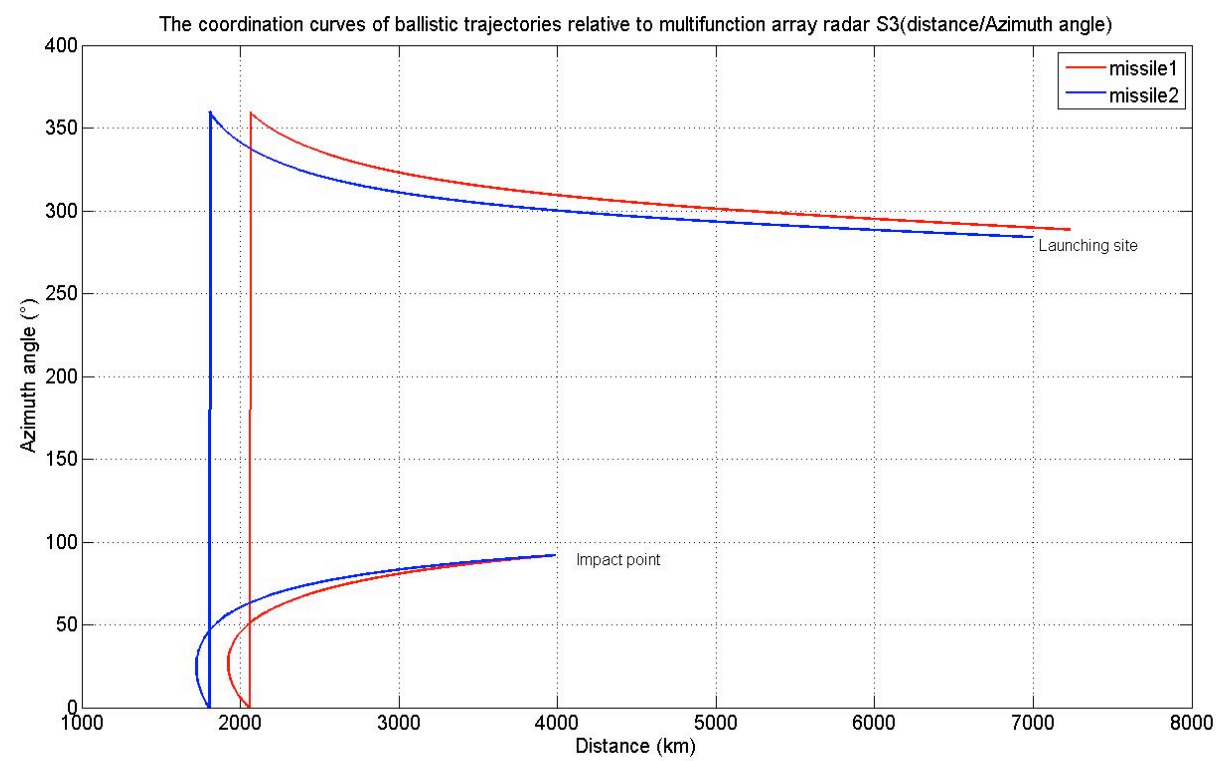

(b) Distance/Azimuth angle

Fig. (12). The coordination curves of ballistic multifunction array radar S3.

Table 2-1. The time-window of detecting and tracking in two modes.

\begin{tabular}{|c|c|c|c|c|c|c|c|c|}
\hline \multirow{2}{*}{ Items } & \multicolumn{2}{|c|}{ DSP } & \multicolumn{2}{|c|}{$\begin{array}{l}\text { Phased-Array Radar } S_{I} \\
\text { (Forward-Deployment) }\end{array}$} & \multicolumn{2}{|c|}{ Distance Early Warning Radar $S_{2}$} & \multicolumn{2}{|c|}{$\begin{array}{l}\text { Multifunction Array } \\
\text { Radar } S_{3}\end{array}$} \\
\hline & Missile 1 & Missile 2 & Missile 1 & Missile 2 & Missile 1 & Missile 2 & Missile 1 & Missile 2 \\
\hline $\begin{array}{l}\text { Time-window in heteroge- } \\
\text { neous MAS model (s) }\end{array}$ & $(75,270)$ & $(80,240)$ & l & l & $\begin{array}{c}(125,475, \\
570)\end{array}$ & $(93,495,650)$ & $\begin{array}{l}(662 \\
1420)\end{array}$ & $(613,1397)$ \\
\hline
\end{tabular}




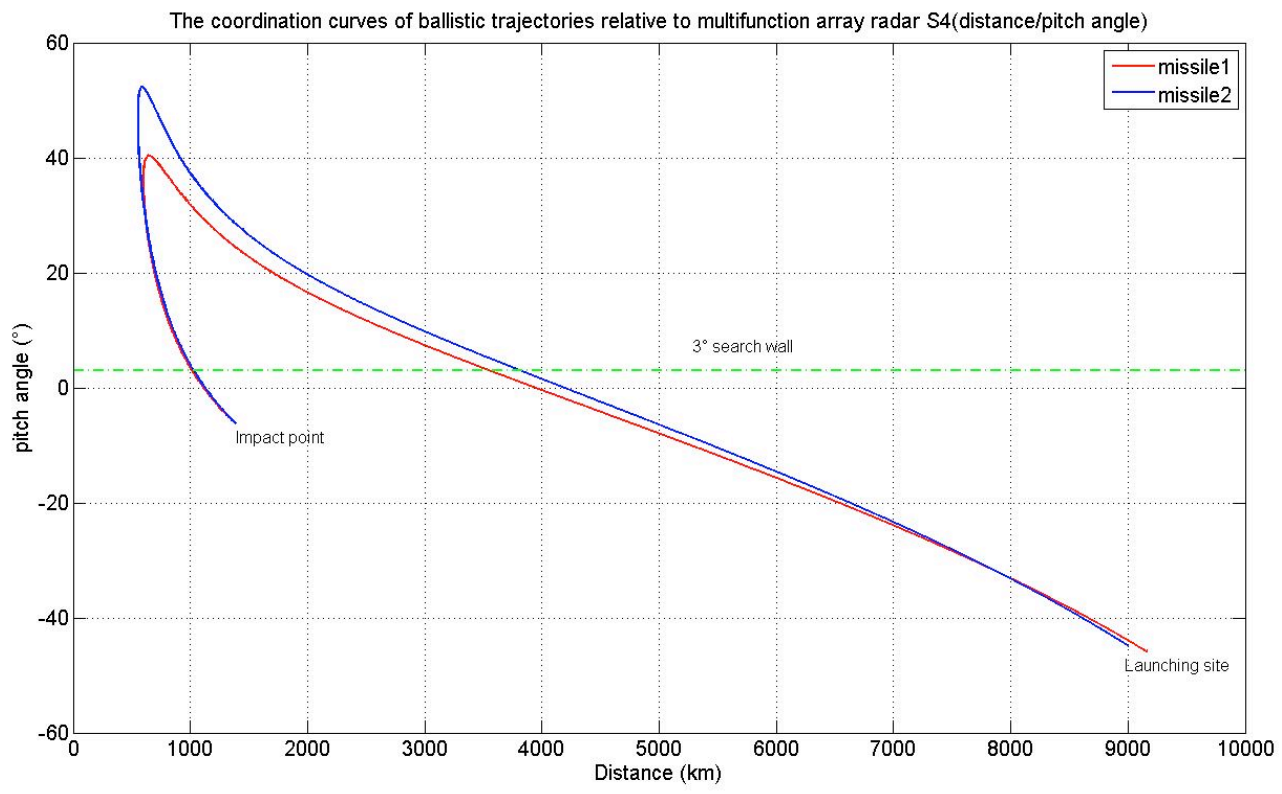

(a) multifunction array radar $S_{4}$

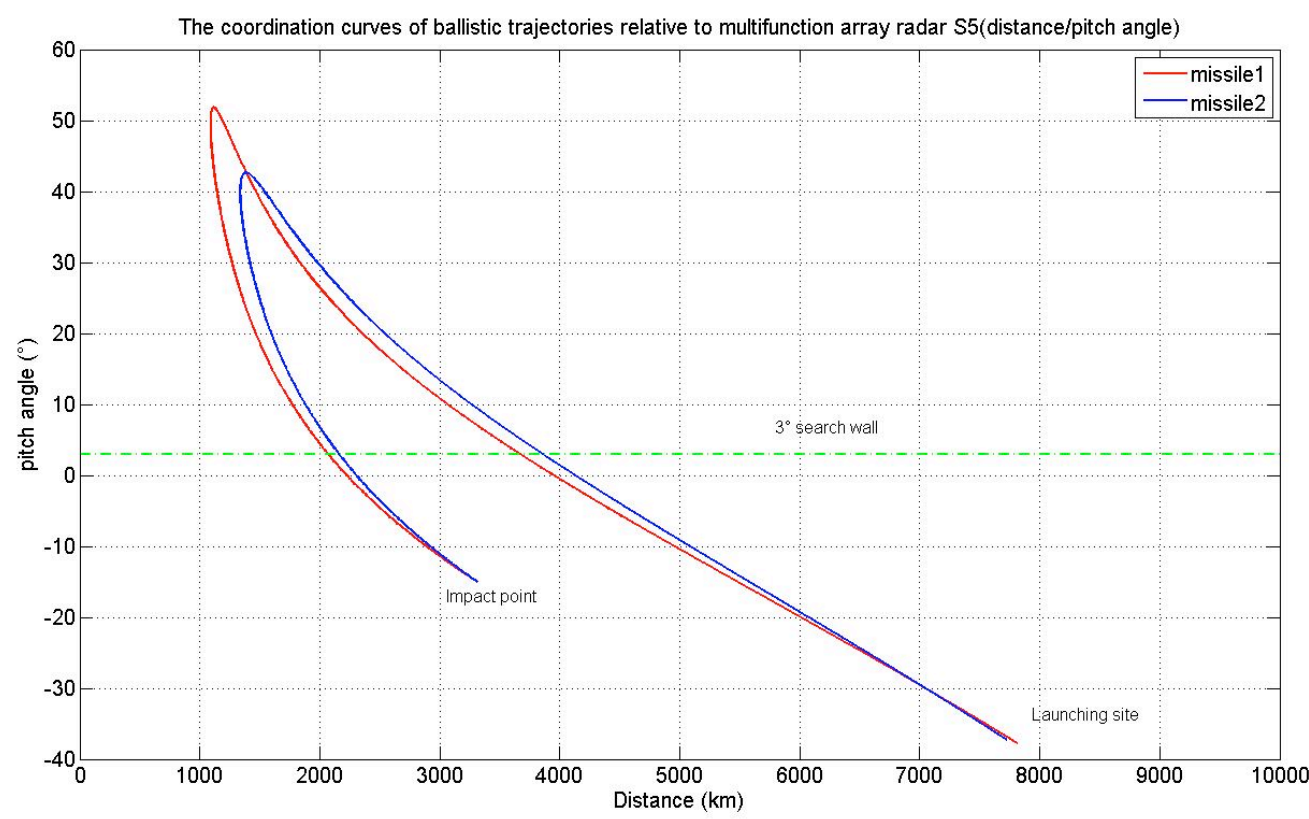

(b) multifunction array radar $S_{5}$

Fig. (13). The distance/pitch angle curves of ballistic trajectories relative to multifunction array radar $S_{4}$ and multifunction array radar $S_{5}$ (Using horizontal search, and the pitch angle of search is set to $3^{\circ}$ ).

Table 2-2. The time-window of detecting and tracking in two modes.

\begin{tabular}{|c|c|c|c|c|c|c|}
\hline \multirow{2}{*}{ Items } & \multicolumn{2}{|c|}{ Multifunction Array Radar $S_{4}$} & \multicolumn{2}{|c|}{ Multifunction Array Radar $S_{5}$} & \multicolumn{2}{|c|}{ The Whole Time-Window of System } \\
\hline & Missile 1 & Missile 2 & Missile 1 & Missile 2 & Missile 1 & Missile 2 \\
\hline $\begin{array}{l}\text { Time-window in heterogene- } \\
\text { ous MAS model (s) }\end{array}$ & $(824,1440)$ & $(857,1435)$ & $(1432,2130)$ & $(1431,2160)$ & $(75,475)(662,2130)$ & $(80,495)(613,2160)$ \\
\hline $\begin{array}{l}\text { Time-window in } \\
\text { tradition model (s) }\end{array}$ & $(860,1440)$ & $(883,1435)$ & $(1537,2130)$ & $(1523,2160)$ & $\begin{array}{c}(75,475)(860,1440) \\
(1537,2130)\end{array}$ & $\begin{array}{c}(80,495)(883,1435) \\
(1523,2160)\end{array}$ \\
\hline
\end{tabular}


The tracking time-window of the system for missile 1

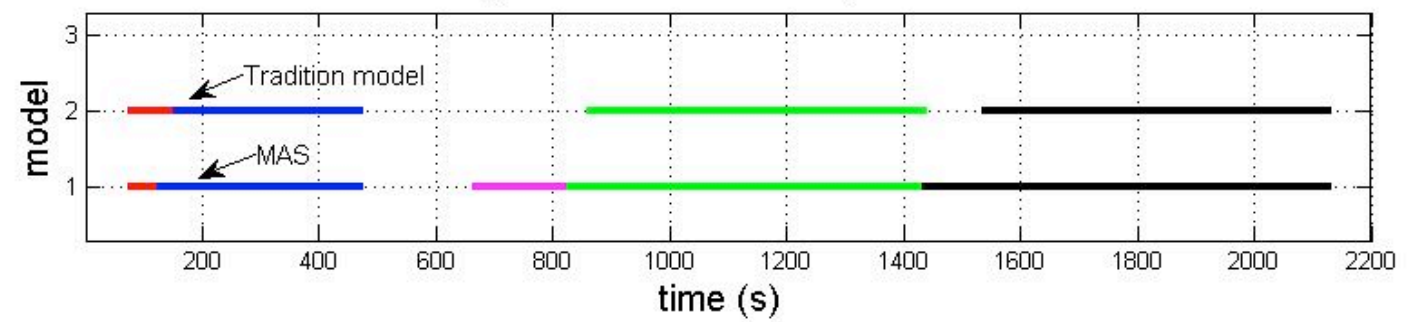

The tracking time-window of the system for missile 2

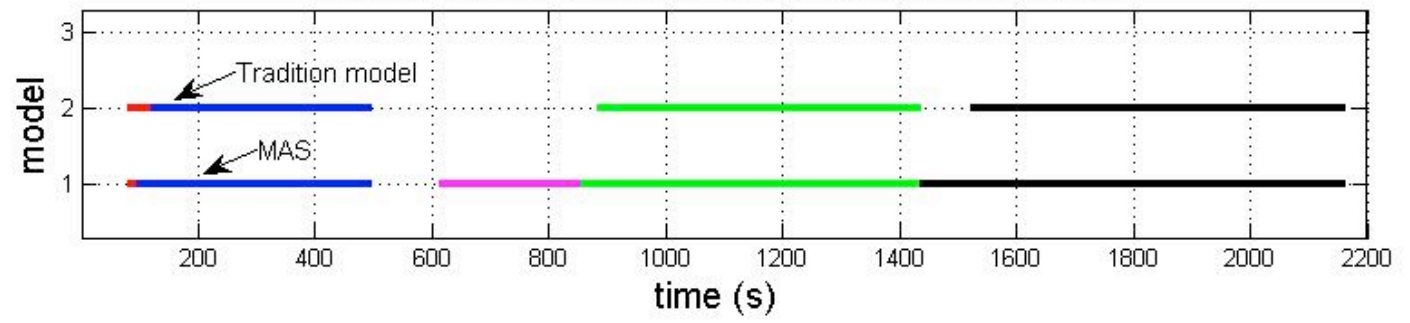

Fig. (14). The tracking time-window of system in two modes.

(Note: The second-bit data of the over striking data denotes that the target exceeds the maximal tracking ability of the radar, and the third-bit data means to fly out of detection area.)

From Table 2, we can see that the early-warning time for two trajectories provided by DSP satellite is $1800 \mathrm{~s}$ and 1900s. The target has a heading process for X-wave radar, and the radar can't effectively trace ballistic targets for a long time, so there is no tracking ability for ballistic targets. Ballistic trajectory is predictable, under heterogeneous MAS system, community of interest rapidly guides long-distance early-warning radar with tracking ability for ballistic targets to intercept and capture trace, which is 20 s earlier compared with that under self-searching mode.

The targets fly out the detection area at 570s and 650s, which requires external accurate guidance information for guiding to intercept acquisition. Under the mechanism of the paper, the target guidance is given to multi-function phased array radar 1 with good deploy position, which is $190 \mathrm{~s} \sim 220 \mathrm{~s}$ earlier to re-intercept trace than that under traditional mode. For ground-based search, setting the angle of pitch under $3^{\circ}$ mode is 50 s earlier to intercept the tracing targets, which effectively guarantees that the early-warning system can intercept the tracing targets in the maximal tracking range, as shown in Fig. (14).

\section{CONCLUSION}

Ballistic battle sensor task planning framework under heterogeneous MAS segments based on task property, not only balances the task execution of each sensor, but also is helpful to differentiate between the regional difference problems caused by multi-source sensor ability. The paper establishes a sensor task planning sequence generation model based on bi-layer programming, which provides a new idea for ballistic battle sensor task planning. Lastly, the paper makes simulation, and verifies the solution flow of the problem and the efficacy of the method.
The following work not only includes considering the targets and constraints which meet the war situation, and establishing multi-target planning model to make the model closer to the characteristics and decision requirements of the battle, but also includes establishing multi-task distributive intelligent collaborative task planning theory and algorithm, for improving the solution efficiency of the algorithm and the accuracy of the solution under uncertain war situation.

\section{CONFLICT OF INTEREST}

The authors confirm that this article content has no conflicts of interest.

\section{ACKNOWLEDGEMENTS}

Declared none.

\section{REFERENCES}

[1] R. Badawy, B. Hirsch, and S. Albayrak, "Agent-based coordination techniques for matching supply and demand in energy networks," Integrated Computer-Aided Engineering, vol. 17, no. 4, pp. 373382, 2010.

[2] Defense SITREP, Navy: "Air and Missile Defense Radar Program on Track Despite PTSS Termination," Defense SITREP, 2013.

[3] J. Li, Z. Zhong, N. Jing, and W. Hu, "Space-air Resources Multiphase Cooperation Task Planning Approach Based on Heterogeneous MAS Model," Acta Aeronautica et Astronautica Sinica, vol. 34, no.7, pp. 1682-1697, 2010.

[4] K. Luo, Y. Li, and W. Jiang, "Modeling of ballistic missile earlywarning simulation system based on UML," Journal of System Simulation, vol. 22, no.4, pp. 845-849, 2010.

[5] X. Liu, Z. Liu, and W. S. Hou, "Improved MOPSO algorithm for multi-objective programming model of weapon-target assignment," Systems Engineering and Electronics, vol. 35, no.2, pp. 326-330, 2013.

[6] L. Li, F. Liu, and Y. Mei, "Anti-TBM fire target matching optimization and APSO solution algorithm," System Engineering and Electronics, vol. 35, no.5, pp. 993-999, 2013.

[7] M. Maleki, H.R. Bahrami, S. Beygi, and M. Kafashan, "Space Modulation With CSI: Constellation Design and performance 
Evaluation," IEEE Transactions on Vehicular Technology, vol. 62, no.4, pp. 1623-1634, 2013.

[8] P. Ni, J. M. Liu, Y, Liu, and Q, Fu, "Research on mission planning for distributed multi-sensors in anti-TBM combat based on multiagent system," Sensor Letters, vol. 12, no. 2, pp. 325-331, 2014.

[9] Y. Qiao, J. Liu, and C. Li, "Modeling of missile detecting system probability," System Engineering and Electronic Technique, vol. 33, no.10, pp. 2244-2248, 2011.

[10] J. Ren, and Q. Xing, "Ballistic early-warning resource scheduling method using self-adaptation probability particle warm algorithm," Journal of AFEU (Natural science), no. 6, 2014.
[11] S. Tang, "Research on generation mechanism of network ballistic system dynamically intercepting alliance," Changsha: National University of Defense Technology, 2011.

[12] X. Zhou, Z. Wu, and H. Wang, "A backward learning particle swarm optimization algorithm," Chinese Journal of Electronics, vol. 41, no.8, pp. 1647-1652, 2013.

[13] G. Zhang and X. Zou, "Deadline Constrained Task Scheduling Based on Standard-PSO in a Hybrid Cloud," Lecture Notes in Computer Science, vol. 28, no.1, pp. 210-217, 2013.

Received: June 16, 2015

Revised: August 10, 2015

Accepted: September 19, 2015

(C) Peng et al.; Licensee Bentham Open.

This is an open access article licensed under the terms of the (https://creativecommons.org/licenses/by/4.0/legalcode), which permits unrestricted, non-commercial use, distribution and reproduction in any medium, provided the work is properly cited. 